Parijsky: We may be able to obtain an independent value of magnetic field from the Faraday rotation method provided we know the following quantities:

1. A measure of the emission from HII radio continuum or from radio absorption measurements.

2. The relative intensity for the components of the $3727 \AA$ line for [OII].

The latter seems to be very difficult.

Davies: The former gives a measure of the product $N_{\mathrm{e}}^{2} L$ integrated over the line of sight and it is very difficult to reconstruct the required product $N_{\mathrm{e}} L$ which is required to derive $\mathrm{H}$ from measurements of Faraday rotation.

van Damme: I read in the abstract: "One of the strongest Zeeman effects was measured in the narrow feature of the Taurus A absorption spectrum".

In connection with the remark of Dr. Kerr to attack instrumental effects, may I ask, can you tell how many times you actually have measured this feature to overcome statistical effects?

Davies: Six independent measurements each with effective integration times of more than $1 \mathrm{hr}$ were used; each showed the same polarization effect.

Bolton: It is quite clear from the signal/noise ratio of the diagrams which Davies has shown and the attacks of the radio astronomers that this result is extremely suspect. This is an extremely difficult experiment and it is doubtful, even if the present results are correct, whether the measurements could be extended to give a general picture of the magnetic field of the Galaxy. Much more promising are the Faraday rotation type measurements which, although involving integration along the line of sight, are not subject to experimental doubts and difficulties.

Davies: Such Faraday rotation experiments will give the direction of the field and will give the magnitude only if we know the distribution of electrons in the line of sight; this latter distribution is extremely difficult to determine.

Parijsky: It is very interesting to compare the field determined from 21 -cm measurement with that obtained with the Faraday rotation technique. Let us consider the Crab nebula as an example. It is quite possible that the Faraday rotation occurs in the interstellar medium and not in the nebula itself. The serious support came from the high-resolution observations at Pulkovo in polarized emission at 3 and $6 \cdot 4 \mathrm{~cm}$. Then it is easy to show that the sign of the magnetic field coincides for both methods.

\title{
34. OBSERVATION AND INTERPRETATION OF OPTICAL AND RADIO POLARIZATION
}

\author{
G. WeSTERHOUT \\ University of Maryland
}

Measurements of polarization of radio emission from the galactic background were started in 1957 and seemed to be partially successful, but so uncertain that not much weight was attached to them. Only in the last two years have such polarization measurements been fully successful. They were carried out by radio astronomers at Leiden, Cambridge, and Sydney. I shall report here on the measurements made by the Leiden group, and draw some general conclusions. The first year of observations was described by Westerhout et al. (1962) and Brouw, Muller, and Tinbergen (1962); the work has been continued since by Muller, Brouw, Tinbergen, and Berkhuysen (1963). The observations made by the Cambridge group are substantially in agreement with the Leiden work (Wielebinski, Shakeshaft, and PaulinyToth 1962), and so are the Sydney observations, on which Dr. Gardner reports in another paper (this volume, paper 35 ). 
The difficulties of measuring the background polarization are considerable. The situation is rather different from that encountered in optical astronomy and in the measurement of radio stars, where comparisons can be made with neighbouring regions to determine instrumental effects.

In an isotropic radiation field, the intensity received by the average radio telescope does not only come from the direction of the main beam, but about one quarter comes from other directions, so-called sidelobes. And since the surroundings of a telescope are at $300^{\circ} \mathrm{K}$, it will pick up part of the thermal emission from the ground. This stray radiation might be polarized, or the sidelobes might admit only polarized radiation of a certain direction.

Thus, typically, if we change the direction of polarization of our feed, we see a sine wave with a certain amplitude wherever we look. Around $400 \mathrm{Mc} / \mathrm{s}$, at Dwingeloo, this spurious signal was about $2^{\circ} \mathrm{K}$. The total background emission was of the order of $40^{\circ} \mathrm{K}$, and thus a degree of polarization of the order of a few per cent gave a signal equal to the spurious radiation.

The optical polarization measurements allow a somewhat better accuracy. There, the spurious polarization is due to passing of the light through optical surfaces in directions not normal to the surface, to unwanted reflections, and to nightsky light. The probable errors in the optical data are of the order of $\pm 0 \cdot 2 \%$ in percentage polarization, as compared to \pm 0.5 to $1 \cdot 0 \%$ in the radio data.

By rotating the feed, a linearly polarized signal will produce a curve depending on the intensity of the polarized component and on the feed angle with respect to the direction of polarization as $I=I_{0} \cos 2 \theta$.

There are two ways in which one can distinguish the real from the spurious polarization. The first is by assuming (1) that the spurious polarization is due to pick-up from the ground and thus dependent on altitude only (if the surroundings are flat and without obstacles) and (2) that on the average the sky polarization is small and random. By making observations all over the sky one is then left with an average polarization, dependent on elevation only, which must be the spurious component.

The second way is by using the ionospheric Faraday rotation to distinguish a signal from outside the atmosphere. At $400 \mathrm{Mc} / \mathrm{s}$, ionospheric Faraday rotation causes the polarization vector to rotate over about $60^{\circ}$ in the course of the night.

Brouw, Muller, and Tinbergen (1962) were able to measure very accurately this Faraday rotation for a number of bright points in the sky and thus not only prove the extraterrestrial nature of the radiation, but also extrapolate to zero ionosphere and find the real polarization angle in a number of points.

By regularly measuring these points throughout the night, they could establish very accurately the total electron density in the Earth's atmosphere (Brouw and Berkhuysen 1962; see also the Cambridge results, Wielebinski and Shakeshaft 1962). They suggest that such observations in fact are extremely well suited for continuous monitoring of the ionosphere.

Following a number of standard points throughout the night is obviously similar to the observation of standard stars for extinction. 
The results obtained up to the end of 1962 are given in Plates 1 and 2. The intensity of the polarized component and the direction of the electric vector are given, together with some of the contours describing the unpolarized background. Three things are immediately obvious. (1) There is some polarized radiation, although very little, over most of the observed part of the sky. (2) There are two or three places where the intensity is fairly high and the vectors show some alignment. (3) There is no correlation with the background contours. The highest degree of polarization found is about $8 \%$; in most places it is below $3 \%$.

The two most interesting regions are those around $l^{\mathrm{II}}=140^{\circ}, b^{\mathrm{II}}=+5^{\circ}$, and around $l^{\mathrm{II}}=0^{\circ}, b^{\mathrm{II}}=+60^{\circ}$. These are the most intense regions, with polarization temperatures up to 6 or $7^{\circ} \mathrm{K}$. In both regions the polarization vectors seem to follow a somewhat regular pattern, suggestive of curved magnetic fields. Neither of the two regions shows unusual background features. The observations cover the entire "spur" of radiation perpendicular to the galactic plane at about $l \mathrm{II}=30^{\circ}$, but there seems to be no correlation of degree and direction of polarization with the unpolarized intensity of the "spur".

If the magnetic fields in the Galaxy were well aligned we would expect a degree of polarization of the order of $50 \%$, taking into account the contribution from thermal emitters and extragalactic radiation. We therefore need to explain the very low degree of polarization observed. Faraday rotation in the interstellar ionized gas will affect the direction of polarization, and, if large and irregular enough, will also decrease the degree of polarization due to smoothing along the line of sight and across the fairly wide $\left(2^{\circ}\right)$ beam. The number of rotations of the electric vector through $360^{\circ}$ can be written as

$$
n=0 \cdot 73 \times 10^{5} \times(408 / f)^{2} \int N H_{/ /} \mathrm{d} s,
$$

where $f$ is the frequency in $\mathrm{Mc} / \mathrm{s}, N$ is the electron density in $\mathrm{cm}^{-3}, H_{/ /}$is the component of the magnetic field parallel to the line of sight in gauss (the sign of $n$ depends on the sign of $H$ ), and $\mathrm{d} s$ is the path length in parsec. If we take $N=0.01 \mathrm{~cm}^{-3}$, $H_{/ /}=10^{-5} \mathrm{G}$ and $\mathrm{d} s=100 \mathrm{pc}$ we have $n=0.73$ rotations at $408 \mathrm{Mc} / \mathrm{s}$. Clearly, even with a regular magnetic field, the normal irregularities in the distribution of $N$ will tend to cause variations of $n$ over the $2^{\circ}$ beam large enough to depolarize most of the radiation from distances further than a few hundred parsecs. The observed polarized radiation must therefore originate close to the Sun.

However, recent measurements of Faraday rotation in extragalactic sources (Gardner, this volume, paper 35), which give the integral $\int N H_{/ /} \mathrm{d} s$ through the whole Galaxy, show that in many places the product $N H_{/ /}$must be considerably smaller than the value $10^{-7}$ used above. If this is generally true, then Faraday rotation is insufficient to explain the small percentage of polarization.

We are then led to the conclusion that irregular magnetic fields must be an important cause. The existence of a few bright regions suggests that these might be nearby clouds of relativistic particles, or a nearby concentration of the magnetic field, seen against the depolarized background. If this is so, however, this nearby field is clearly not aligned parallel to the galactic plane, at least not over large areas. 
The question now arises why then the optical polarization in some places is so excellently aligned parallel to the galactic plane. Figure 1 illustrates the discrepancy between optical and radio polarization in the bright region around $l=140^{\circ}$, $b^{\mathrm{II}}=+5^{\circ}$. In comparing the two, it should be borne in mind that the optical vectors, if the Davis-Greenstein theory holds, are parallel to the component of the magnetic field normal to the line of sight, whereas the radio vectors are perpendicular. The figure shows some agreement but one can certainly not conclude that it presents a

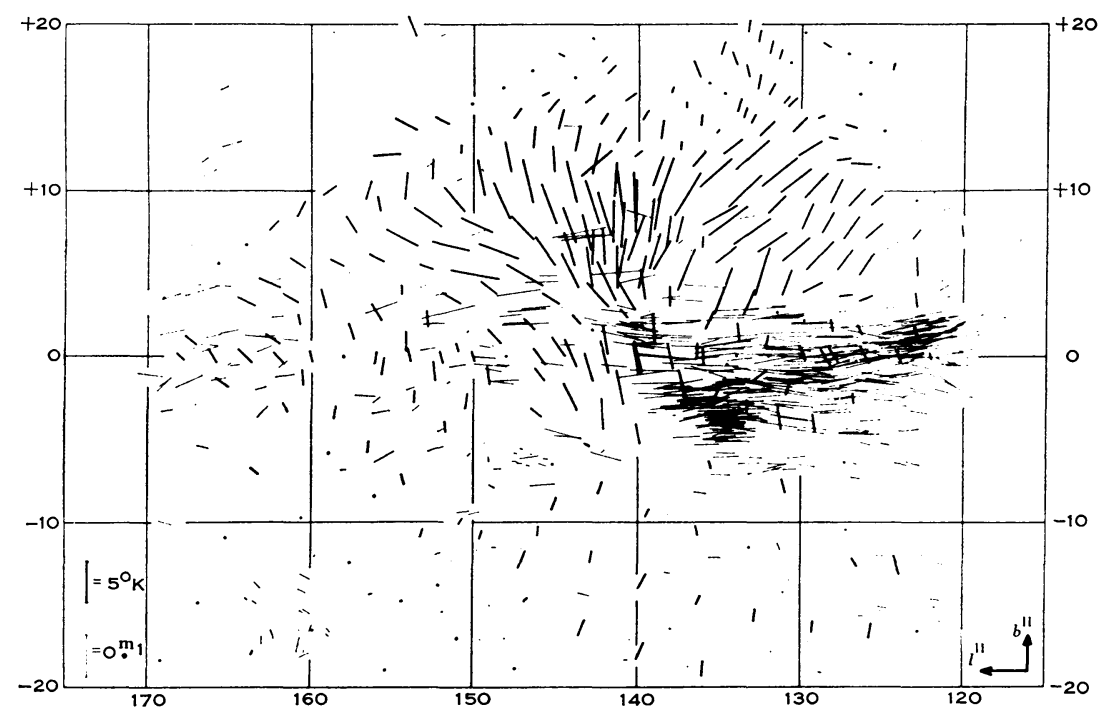

Fig. 1.-Enlargement of part of Plate 2. Thick lines are radio polarization, thin lines are optical polarization (Hall 1958; Behr 1959). The position angle of the lines is that of the electric vector. The Davis-Greenstein mechanism predicts optical electric vectors parallel to the magnetic field. Radio electric vectors are perpendicular to the field if corrected for interstellar Faraday rotation.

clear-cut case. And at somewhat larger distances from the centre of the picture, agreement is lacking altogether. Clearly, though, many more optical polarization measurements are needed before we can make firm statements about agreement or disagreement with the radio data. Also, the radio data should be corrected for interstellar Faraday rotation, which is possible by making observations at a few different frequencies.

There is a weak correlation between absorption and degree of polarization of the starlight. However, the more distant stars, with higher absorption, do not show an increase in polarization. Moreover, in regions such as the one in Figure 1, the direction of polarization is independent of the distance of the stars. These data seem to indicate that the optical polarization, at least in the region of Figure 1, occurs in the solar neighbourhood, perhaps within a few hundred parsecs or so (Hall 1958). Thus, both radio and optical polarization seem to originate in a small region around the Sun; radiation from more distant regions would be depolarized owing to irregularities in the magnetic field. The disagreement in most regions, and 
OBSERVATION AND INTERPRETATION OF OPTICAL AND RADIO POLARIZATION

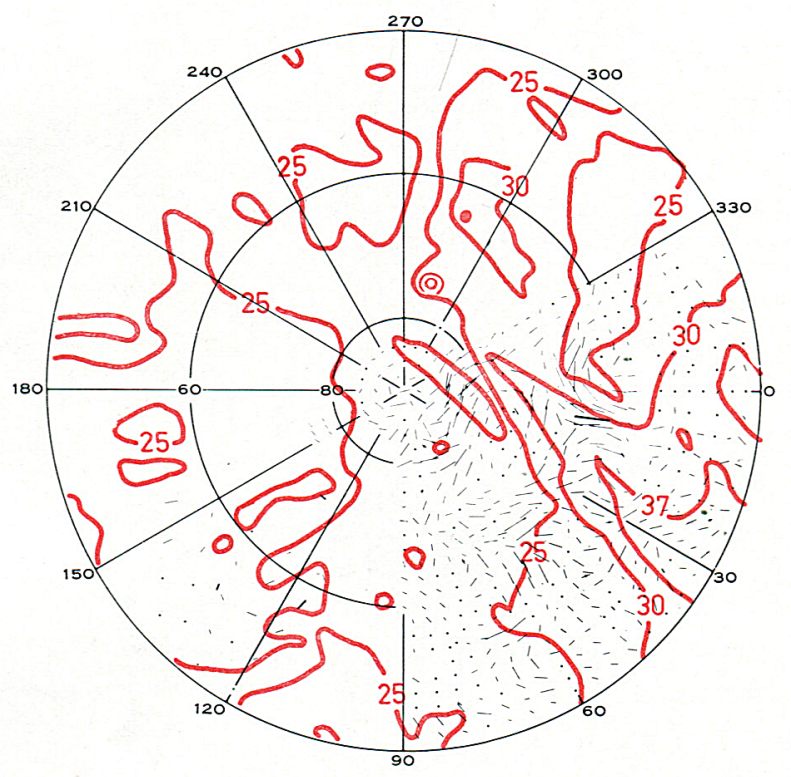

Polarization measurements at $408 \mathrm{Mc} / \mathrm{s}$; all Dwingeloo measurements up to the end of 1962 are combined in this Plate and Plate 2. The length and direction of the lines indicate the intensity and the plane of the electric vector. In the lower left-hand corner of Plate 2 the intensity scale is given. The contours give a rough indication of the randomly polarized background, and are labelled in ${ }^{\circ} \mathbf{K}$ of brightness temperature.

The maps are in new galactic coordinates. 
OBSERVATION AND INTERPRETATION OF OPTICAL AND RADIO POLARIZATION

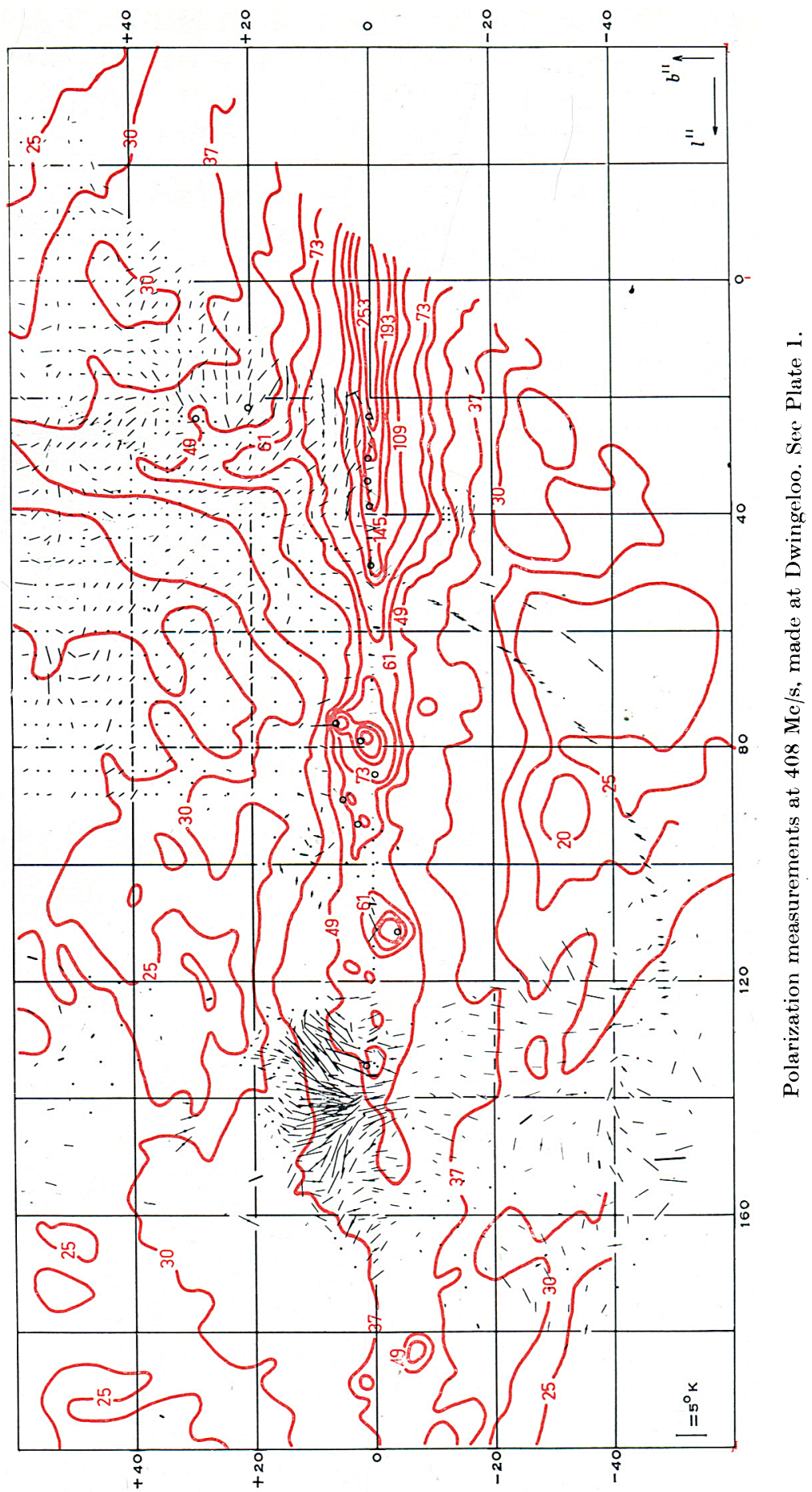

IAU-LRSI Symp. Iol. 20, Paper 34 
agreement in some, between optical and radio polarization directions could perhaps be explained partly by Faraday rotation, partly by the fact that optical absorbers and nonthermal radio emitters do not occur in the same regions of space.

The one important conclusion one can draw from the above is that the radio data do not seem to be representative of a general magnetic field. This then raises the question whether the galactic nonthermal background is really a. smooth background or consists of a large number of extended discrete sources. The few regions where the polarized intensity is large would then be the nearest ones of these sources. The sources could have their own magnetic field and perhaps also contain a concentration of relativistic electrons. In directions where the line of sight through the Galaxy is very long, the background radiation would be smooth. In regions like the anticentre, however, one might expect the smooth background to break up into individual pieces. This seems indeed to have been observed (R. D. Davies, private communication). Also, at least part of the so-called "galactic halo" radiation could well be due to nearby emitters; it shows a considerable amount of structure.

On the basis of present evidence, therefore, one should consider the possibility that the magnetic field is not a smooth field following the spiral arms, but perhaps a broken-up field. One is tempted to consider this field as a result of old supernova explosions, the optical remnants of which have long since disappeared, but of which the radio emission is still appreciable, either by stronger radiation from the smoothly distributed relativistic electrons, or by radiation from its own electrons. Such a model would be consistent with the optical and radio observations of polarization, and with the observations of the continuous background emission.

\section{References}

BeHr, A. (1959).-Nachr. d. Akad. d. Wiss. Göttingen, math.-phys. Klasse Nr 7: 185-240.

Brouw, W. N., and Berkhuysen, E. M. (1962).-Nature 196: 757-78.

Brouw, W. N., Muller, C. A., and Tinbergen, J. (1962).-B.A.N. 16: 213-24.

HALL, J. S. (1958).-Pub. U.S. Naval Obs. 17 : 273-342.

Muller, C. A., Berkhuysen, E. M., Brouw, W. N., and Tinbergen, J. (1963).-Nature 200: 155-6.

Westerhout, G., Seeger, C. L., Brouw, W. N., and Tinbergen, J. (1962).-B.A.N. $16: 187-212$.

Wielebinski, R., Shakeshaft, J. R., and Pauliny-Toth, I. I. K. (1962).-Observatory 82 : 158-64.

Wielebinski, R., and Shakeshaft, J. R. (1962).-Nature 195: 982-3.

\section{RADIO POLARIZATION EFFECTS IN THE GALAXY}

\section{F. F. GARDNer}

\section{CSIRO Radiophysics Laboratory, Sydney}

Polarization effects involving our Galaxy have been studied in two ways : firstly, by observations of the polarization of the galactic radiation itself, and secondly, by the effects of galactic magnetic fields and ionization on the polarization of extragalactic radio sources. For an aerial of fixed dimensions, the intensity of the background varies at about $\lambda^{2 \cdot 5}$ ( $\lambda$ is wavelength) and that of the sources at about $\lambda^{0 \cdot 7}$. Thus background measurements are restricted to the longer wavelengths where the 